\title{
Impacto de las actividades físicas y recreativas para disminuir la depresión en el adulto mayor
}

\author{
Impact of Physical and Recreational Activities to reduce Depression in the Elderly
}

\author{
Margarita Lazcano-Ortiz ${ }^{a}$, Maria L Sánchez-Padilla, ${ }^{b}$ Reyna C. Jiménez-Sánchez ${ }^{c}$, Gabriela \\ Banda-Almazán ${ }^{d}$, Paola A. Guerrero-Jiménez ${ }^{d}$, Hernández Hernández Cristhian-Efrén ${ }^{d}$ \\ Sánchez Juárez Carolina ${ }^{d}$
}

\begin{abstract}
:
Objective: To know the impact of physical and recreational activities to reduce depression in older adults living in the House of the Third Age of Pachuca de Soto, Hgo. Material and methods: Quasi-experimental type research. A sample of 6 older adults, through pre-post of a program of activities with 13 sessions lasting 5.5 hours a week. Two instruments were applied; a questionnaire of 20 items and the Scale of Yesavage. Results: The average age was 74.17 years $(S D=6.274,66-80)$. Activities with greater interest, walking and dancing; which managed to eliminate depression in $50 \%$ of the participants, demonstrating a favorable impact. Conclusions: Through the implementation of physical and recreational activities chosen by the elderly, a decrease in the level of depression was generated.
\end{abstract}

Keywords:

Older adult, depression, physical activities, recreational activities

\section{Resumen}

Objetivo: Conocer el impacto de las actividades físicas y recreativas para disminuir la depresión en los adultos mayores residentes en Casa de la Tercera Edad de Pachuca de Soto, Hgo. Material y métodos: Investigación de tipo cuasiexperimental. Una muestra de 6 adultos mayores, a través de un pre-test y post-test de un programa de actividades con 13 sesiones con una duración de 5.5 horas a la semana. Se aplicaron dos instrumentos; un cuestionario de 20 ítems y la Escala de Yesavage. Resultados: El promedio de edad fue de 74.17 años ( $\mathrm{DE}=6.274$; 66-80). Actividades con mayor interés, caminata y baile; las cuales lograron eliminar la depresión en un $50 \%$ de los participantes, demostrando un impacto favorable. Conclusiones: A través de la implementación de actividades físicas y recreativas elegidas por los adultos mayores se generó una disminución del nivel de depresión por el que cursaban.

Palabras Clave:

Adulto mayor, depresión, actividades físicas, actividades recreativas

a Autor de Correspondencia, Profesor Investigador Universidad Autónoma del Estado de Hidalgo, Instituto de Ciencias de la Salud, Área Académica de Enfermería ORCID: 0000-0002-9653-9291,Pachuca Hgo. México. Email: margarita_lazcano@uaeh.edu.mx

${ }^{\text {b }}$ Profesor investigador .Universidad Autónoma del Estado de Hidalgo, Instituto de Ciencias de la Salud, Área académica de Enfermería. Pachuca Hgo. México. Email: marialuisasanchezpadilla@yahoo.com.mx

c Profesor investigador .Universidad Autónoma del Estado de Hidalgo, Instituto de Ciencias de la Salud, Área académica de Enfermería. Pachuca Hgo. México. Email: Cristyji@ hotmail.com

d Alumnos de Licenciatura en Enfermería de la Universidad Autónoma del Estado de Hidalgo, Instituto de Ciencias de la Salud, Área académica de Enfermería. Pachuca Hgo. México. efrenhudson4@gmail.com 


\section{Introducción}

La depresión se presenta como un conjunto de síntomas de predominio afectivo (tristeza patológica, apatía, desesperanza, decaimiento, irritabilidad, sensación subjetiva de malestar e impotencia frente a las exigencias de la vida) aunque, en mayor o menor grado, también están presentes síntomas de tipo cognitivo, volitivo y somático, por lo que podría hablarse de una afectación global psíquica y física, haciendo especial énfasis en la esfera afectiva [1]

Hipócrates emplea el término genérico de "melancolía" para dar cuenta de estos estados de inhibición y tristeza que, por cierto, Aristóteles (siglo IV a. C.) aporta en algunos escritos para relacionarlos con personas especialmente sensibles e inteligentes. Para Hipócrates, la melancolía se debe a desequilibrios en la secreción de bilis negra, o bien a una mala combustión de esta sustancia dentro del organismo que daría lugar a restos tóxicos; de hecho, el termino proviene del griego melaina chole (bilis negra).

Kraepelin (1896) reservo el término de "melancolía" para referirse a las depresiones de la familia, su curso y su gravedad: los maniacodepresivos incluían cuadros que hoy denominaríamos depresión mayor, distimia, trastorno bipolar o ciclotimia, los cuales tendrían una mayor historia de antecedentes de enfermedad, menor gravedad y un curso menos crónico que el de las personas con esquizofrenia.

Esta concepción humoral de los trastornos mentales se conservará prácticamente intacta en la medicina occidental hasta el siglo XIX. Aunque el término melancolía era la etiqueta diagnostica más usada, la palabra "depresión" se comienza a utilizar con frecuencia durante el siglo XIX, y ya a principios del siglo $X X$ se emplea como termino diagnostico con bastante asiduidad.

Instituto Nacional de la Salud Mental [2] menciona que no existe una única causa conocida de la depresión. Más bien, parece ser el resultado de una combinación de factores genéticos, bioquímicos y psicológicos. Investigaciones indican que las enfermedades depresivas son trastornos del cerebro. Las nuevas tecnologías que han hecho posible obtener imágenes del cerebro tales como las imágenes por resonancia magnética, han demostrado que el cerebro de las personas con depresión luce diferente del de quienes no la padecen. Las áreas del cerebro responsables de la regulación del ánimo, pensamiento, apetito y comportamiento parecen no funcionar con normalidad

La depresión no es algo normal en el proceso de envejecimiento y la mayoría de los adultos mayores se sienten satisfechos con sus vidas, a pesar del aumento de dolores físicos Sin embargo, cuando la depresión se presenta en adultos mayores, puede que no se le brinde la adecuada atención porque en la vejez se muestran síntomas menos evidentes y ya que se encuentran menos propensos a sufrir o reconocer, sentimientos de pena o tristeza [3].

Es comúnmente un problema de salud mental en los adultos mayores. Un $15 \%$ de los adultos mayores de más de 65 años sufren este trastorno y muchas veces no está diagnosticado o tratado como debiera. Sólo $15 \%$ de los adultos mayores con depresión reciben un tratamiento adecuado [4] De los adultos mayores que están restringidos a sus domicilios, entre $26 \%$ y $44 \%$ tienen depresión y se estima que de $30 \%$ a $50 \%$ de todos los adultos mayores tendrán un episodio de depresión durante el transcurso de sus vidas [5]. Por lo que el propósito es conocer el impacto de las actividades físicas y recreativas para disminuir la depresión en el adulto mayor.

\section{Material y métodos}

Se realizó un estudio de intervención en una población de 20 adultos mayores residentes de la casa de la tercera edad, perteneciente a Pachuca de Soto, Hidalgo. De los cuales se obtuvo una muestra de 6 participantes mediante la aplicación de un pre-test (Escala de depresión geriátrica de Yesavage, que determino un nivel de depresión acorde a los criterios de inclusión del estudio, mismos a los que se les realizó el cuestionario de depresión en el adulto mayor, que consta de 20 ítems, para obtener datos generales y que determina algunos de los factores que influyen de forma negativa en el estado de ánimo, así como características físicas, patológicas y/o psicológicas que limitan la realización de actividades físicas y/o recreativas que pudieran disminuir el nivel de depresión en ellos y de esta manera implementarlas en las sesiones de intervención.[6]

Se realizaron dichas actividades en un total de 13 sesiones llevadas a cabo del 23 de marzo al 20 de abril de 2018, realizando la intervención tres días a la semana, siendo partícipes los investigadores y los colaboradores por parte de la Casa de la Tercera Edad; los días lunes las sesiones tuvieron una duración de una hora, los días miércoles de una hora con treinta minutos y los días viernes una duración de tres horas. Al finalizar la intervención, se aplicó un post-test (Escala de Yesavage), el cual determinó el cambio en el nivel de depresión. El instrumento utilizado en este estudio obtuvo una puntuación de 0.76 de acuerdo con el Coeficiente de Confiabilidad Kuder Richardson. 
Tabla 1: Nivel de Depresión

\begin{tabular}{cccccc}
\hline $\begin{array}{c}\text { Pre-Test } \\
\text { Nivel de } \\
\text { depresión }\end{array}$ & Fr & $\%$ & $\begin{array}{c}\text { Post-Test } \\
\text { Nivel de } \\
\text { depresión }\end{array}$ & Fr & $\%$ \\
\hline $\begin{array}{c}\text { Sin } \\
\text { Depresión }\end{array}$ & 0 & 0 & $\begin{array}{c}\text { Sin } \\
\text { Depresión }\end{array}$ & 3 & 50 \\
$\begin{array}{c}\text { Depresión } \\
\text { moderada }\end{array}$ & 4 & 66.70 & $\begin{array}{c}\text { Depresión } \\
\text { Moderada }\end{array}$ & 2 & 33.30 \\
$\begin{array}{c}\text { Depresión } \\
\text { Severa }\end{array}$ & 2 & 33.30 & $\begin{array}{c}\text { Depresión } \\
\text { Severa }\end{array}$ & 1 & 16.70 \\
Total & 6 & 100 & Total & 6 & 100 \\
\hline
\end{tabular}

Fuente: Escala de Yesavage

\section{Resultados}

Los resultados que se obtuvieron en los adultos mayores fue el promedio de edad con 74.17 años, (DE= $6.274 ; 66-80$ ), el género que predomina es masculino $(67 \%)$, estado civil de mayor frecuencia es unión libre y viudo $(33 \%)$

Se puede observar en la tabla 1 que el nivel de depresión en el pre-test de 4 personas $(66.70 \%)$ con depresión moderada y 2 (33.30\%) con depresión severa, mientras que en el post-test se eliminó el grado de depresión en 3 personas (50\%) $2(33.30 \%$ ) y tan solo $1(16.70 \%)$ se mantuvo con depresión severa.

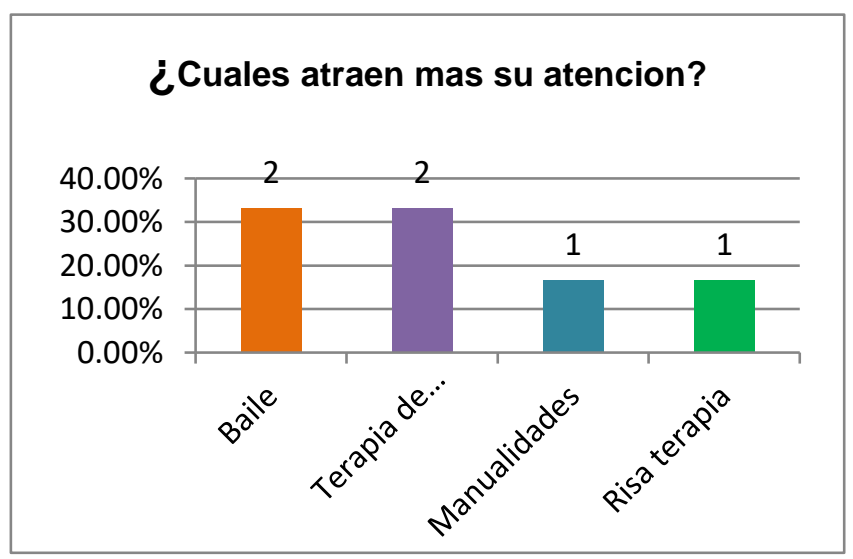

Figura 1. Actividades recreativas

Se observa en la figura 1 que el $88.30 \%$ considera que sí mejoró su estado de ánimo con las actividades realizadas.

En la Figura 2 se observa que dentro de las actividades físicas el $50 \%$ prefirió la caminata.
Figura 2. Actividades que atraen su atención

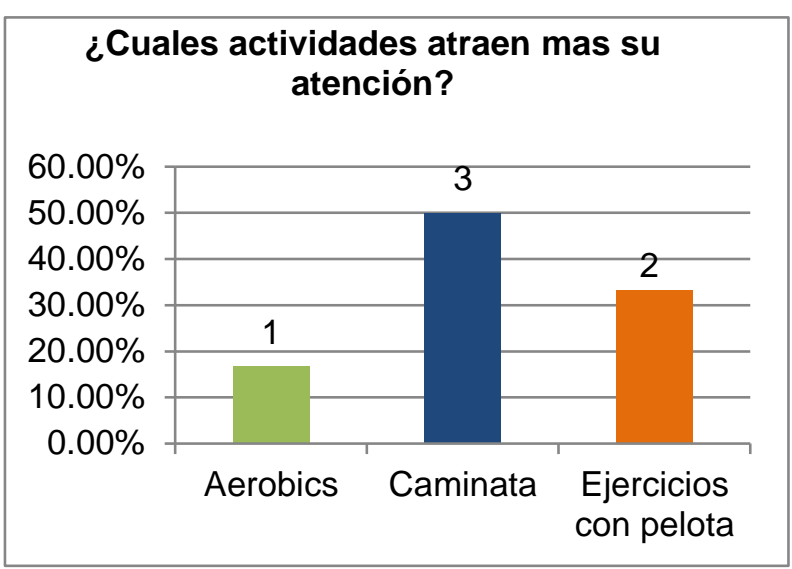

En la Figura 3 se observa que las actividades recreativas de mayor interés fueron baile y terapia de olores, con el $33.30 \%$ cada una.

Figura 3. Nuevas actividades

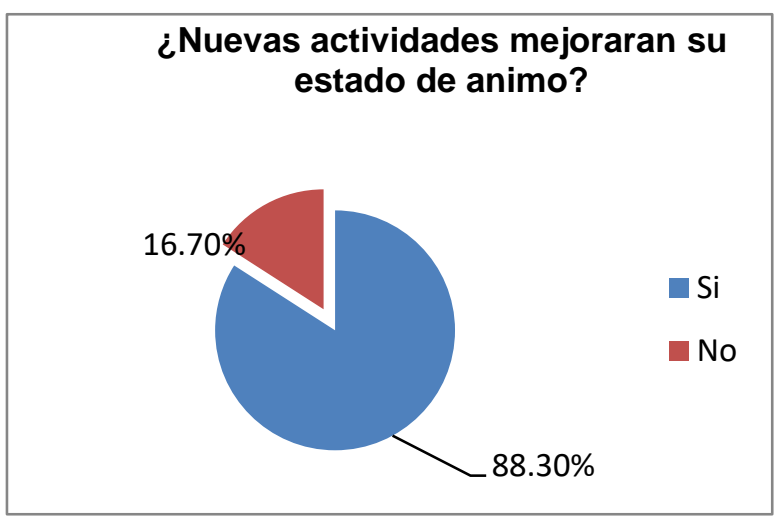

\section{Discusión y conclusiones}

La depresión en el adulto mayor, en términos de prevalencia, es cada vez mayor; pero además es una enfermedad que provoca una importante incapacidad, lo que facilita la presencia de nuevas patologías o el agravamiento de las ya existentes. En la actualidad la depresión se considera un problema de salud pública con graves consecuencias de discapacidad [7]. De aquí la importancia de su detección temprana para un tratamiento oportuno y eficaz, mediante acciones y/o programas destinados a la población vulnerable como lo son los adultos mayores en su estado anímico es fundamental para que interactúe y participe en las actividades propias de la familia, la sociedad y el medio donde se desarrolle. [8] 
No es propiamente el proceso de envejecimiento el que se relaciona con síntomas depresivos, sino que puede derivarse de diversas circunstancias asociadas como la pérdida de la salud, la pérdida de las amistades y una reducción importante de los ingresos

En comparación al estudio: Impacto de un Programa de Psicología Positiva en Sintomatología Depresiva y Satisfacción Vital en Adultos Mayores [9] se demostró que los niveles de depresión disminuyeron significativamente en el grupo de participes mediante actividades físicas y recreativas, por lo que se encuentra una similitud en la investigación.

La atención del adulto mayor debe abordarse con una visión multidimensional tomando en cuenta elementos económico-sociales, biológicos, culturales y familiares que le ayuden a incrementar positivamente su estado de bienestar. [10]

Es esencial implementar programas multidisciplinarios para el abordaje integral de los adultos mayores que puedan atender diferentes aspectos como su nutrición, tratamiento médico, pero también actividades físicas y recreativas como la música, el baile, juegos de mesa, manualidades, actividades que incrementen sus estados de felicidad y eviten algún estado de depresión [11]

La mayor limitante de esta investigación fue el corto periodo de intervención, debido a esto se obtuvieron resultados favorables en sólo el $50 \%$ de los participantes, por lo cual se sugiere dar seguimiento al presente estudio y alargar el tiempo de intervención para comprobar de manera concisa el impacto que las actividades físicas y recreativas tienen en la disminución del nivel de depresión en el adulto mayor.

\section{Referencias}

[1] National Collaborating Centre for Mental Health. Depression: The Treatment and Management of Depression in Adults: [en línea]. 2010. [Fecha de acceso 28 de febrero de 2018]. URL disponible en: https://www.ncbi.nlm.nih.gov/pubmed/22132433

[2] Instituto Nacional de la Salud Mental. Depresión. [en línea]. 2009. [Fecha de acceso 09 de marzo de 2018]. URL disponible en: https://www.nimh.nih.gov/health/publications/espanol/depresion/depres ion_38791.pdf

[3] Justino, L., Bertoldo, T., Zarpellon, G. Influencia del Ejercicio Físico en los Sintomas Depresivos y en la Aptitud Funcional de Ancianos en el Sur de Brasil. [en línea]. 2010. [Fecha de acceso 15 de abril 2018]. URL disponible en : http://www.elsevier.es/es-revista-revista-espanolageriatria-gerontologia-124-articulo-influencia-del-ejercicio-fisico-losS0211139X09003291

[4] Lazcano, M., Sánchez, ML., Lázaro, A., García, ME., Piedra, A, Gayosso, E. Nivel de depresión en el adulto mayor. [en línea]. 2015. [Fecha de acceso 03 de marzo de 2018]. URL disponible en https://www.uaeh.edu.mx/scige/boletin/icsa/n7/p30.html
[5] Del Milagro López I. \& María Rodríguez Hernández H. Propuesta de intervención para ancianos deprimidos. Revista Cubana Med Gen Integr. 1999; 15(1) 19-23

[6] Calero, S., Klever, T., Caiza, M., Rodríguez, AF. \& Analuiza, EF. Influencia de las Actividades Físico-recreativas en la Autoestima del Adulto Mayor. Revista Cubana de Investigaciones Biomédicas. 2016. 35(4): 366-374

[7] OMS. Depresión. [en línea] 2011. [Fecha de acceso 26 de mayo del 2018]. URL disponible en: http://www.who.int/mental_health/management/depression/es/

[8] Araníbar, P. Acercamiento conceptual a la situación del adulto mayor en América Latina. [en línea]. 2001. [Fecha de acceso 26 de abril del 2018]. URL disponible en: http://www.facso.uchile.cl/observa/acercamiento\%20conceptual $\% 20 \mathrm{a} \%$ 201 \% 20 situación\%20del\%20adulto\%20mayor\%20en\%20america\%201 atina.pdf

[9] Cuadra, A., Veloso, C., Puddú, G., Salgado, P. \& Peralta, J. Impacto de un programa de psicología positiva en sintomatologia depresiva y satisfacción vital en adultos mayores. Revista Psicologia: Reflexão e Crítica. Universidade Federal do Rio Grande do Sul Porto Alegre, Brasil 2012. 25(4): 644-652.

[10]Vázquez, C., Sanz., J. Trastorno del Estado de Ánimo: Aspectos Clínicos. [en línea]. 2008. [Fecha de acceso 25 de abril de 2018]. URL disponible en: http://webs.ucm.es/info/psisalud/carmelo/PUBLICACIONES_pdf/2008

-Trastornos_estado_animo_I\%20_Vazquez_y_Sanz_pd

[11] Justino, L., Bertoldo, T., Zarpellon, G. Influencia del Ejercicio Físico en los Síntomas Depresivos y en la Aptitud Funcional de Ancianos en el Sur de Brasil. [en línea]. 2010. [Fecha de acceso 15 de abril 2018]. URL disponible en : http://www.elsevier.es/es-revista-revista-espanolageriatria-gerontologia-124-articulo-influencia-del-ejercicio-fisico-losS0211139X09003291 